\title{
Scolia
}

Revue de linguistique

\section{La topicalisation en français et en norvégien}

Topicalization in French and Norwegian

Hans Petter Helland, Christine Meklenborg Nilsen et Terje Lohndal

\section{(Q) OpenEdition}

Journals

Édition électronique

URL : http://journals.openedition.org/scolia/1176

DOI : $10.4000 /$ scolia. 1176

ISSN : 2677-4224

Éditeur

Presses universitaires de Strasbourg

Édition imprimée

Date de publication : 10 juillet 2020

Pagination : 51-69

ISBN : 979-10-344-0067-6

ISSN : 1253-9708

Référence électronique

Hans Petter Helland, Christine Meklenborg Nilsen et Terje Lohndal, « La topicalisation en français et en norvégien », Scolia [En ligne], 34 | 2020, mis en ligne le 10 juillet 2020, consulté le 10 juillet 2020. URL http://journals.openedition.org/scolia/1176 ; DOI : https://doi.org/10.4000/scolia.1176 
SCOLIA 34/ 2020, p. 51-69.

\title{
La topicalisation en français et en norvégien
}

\author{
Hans Petter HELLAND \\ Université d'Oslo \\ h.p.helland@ilos.uio.no
}

Christine MekLenborg Nilsen

Université d'Oslo

c.m.salvesen@ilos.uio.no

Terje LOHNDAL

NTNU, Trondheim \& Université de Tromsø

terje.lohndal@ntnu.no

\section{Introduction}

Cet article se propose d'étudier la topicalisation dans deux langues typologiquement distinctes, en l'occurrence le français, langue romane, et le norvégien, langue germanique. A partir de notre analyse de la dislocation comme procédé de topicalisation, nous verrons qu'il y a d'importantes différences entre les deux langues en ce qui concerne l'élaboration de la périphérie gauche de la phrase.

Telle sera notre démarche: nous commencerons par une illustration des différences entre les deux langues au niveau du marquage de la périphérie gauche de la phrase. Sur la base d'un ensemble de considérations théoriques, nous proposerons dans la section 2 un examen détaillé des deux systèmes, français et norvégien. Nous signalerons dans la section 3 un certain nombre d'implications théoriques en insistant sur l'interface entre la syntaxe et la pragmatique sous forme d'une approche cartographique. 


\section{Différences structurales entre le français et le norvégien}

\subsection{Langues SVO et Verbe second (V2)}

Contrairement au français, le norvégien est une langue V2; autrement dit, dans une phrase indépendante le verbe doit apparaître comme le deuxième constituant de la phrase (Holmberg, 2015). De telles différences sont illustrées par les phrases (1) et (2):

1) Ce matin, j'ai perdu mon sac.

2) I dag morges mistet jeg sekken min.

à jour matin perdre.PRET je sac.DEF mon

Ce matin, j'ai perdu mon sac.

Dans la phrase française en (1), l'adverbial antéposé ce matin précède immédiatement le sujet avec le verbe comme troisième constituant (V3), alors que son équivalent norvégien (2) illustre la contrainte V2 avec le verbe mistet "perdis" qui suit obligatoirement l'adverbial $i$ dag morges "ce matin» en position initiale. En adoptant les principes de la grammaire générative (Pollock, 1989, 1997; Rowlett, 2007 parmi d'autres), nous dirons que le verbe fini du français atterrit obligatoirement dans la position T (pour Tense - «Temps»), ce qui n'est pas le cas pour le norvégien où le verbe monte d'abord sous $\mathrm{T}$, et ensuite sous la projection CP complementizer phrase - "groupe complémenteur" laissant le sujet comme spécifieur du TP Tense phrase - «groupe temporel». Il s'ensuit que la syntaxe de la phrase, qu'il s'agisse du français ou du norvégien, est fondamentalement liée à l'élaboration de sa périphérie gauche, mais qu'il existe des contraintes distinctes qui régissent la syntaxe de langues V2, comme le norvégien, et des langues non-V2 comme le français. Nous obtenons pour (1) et (2) les dérivations simplifiées et provisoires données en (1a) et (1b):

1a) [ ${ }_{\mathrm{CP}}\left[\mathrm{Ce}\right.$ matin] $\left[_{\mathrm{C} 0}\left[_{\mathrm{TP}}\left[\mathrm{j}^{\prime}\right]\left[_{\mathrm{T} 0}\right.\right.\right.$ ai $\left[_{\mathrm{vP}}[\mathrm{je}] \mathrm{V}_{\mathrm{V} 0}\right.$ air $\left[_{\mathrm{VP}}\left[_{\mathrm{V} 0}\right.\right.$ perdu [DP mon sac ]]]]]]]]]

1b) [CP [I dag] [Co mistet [TP [jeg] [To mistet [VP [jeg] [V0 miste [VP [V0 miste [DP sekken min]]]]]]]]].

Nous reviendrons dans ce qui suit (section 3) sur la structuration plus précise de la périphérie gauche de la phrase et comme exemple, nous nous baserons sur les mécanismes de la dislocation. 


\subsection{Dislocation et topicalisation}

Conformément aux principes de la dislocation (Berrendonner, 2015; Delais-Roussarie et al., 2004), un constituant de la phrase peut être repris - dislocation à droite (5) - ou annoncé - dislocation à gauche (6) - hors des limites de la phrase:

3) Je liai perdu ce matin, mon sac $_{i}$.

4) Mon sac $c_{i}$, je líai perdu ce matin.

Le pronom clitique objet ( $\left.l^{\prime}\right)$ est attaché au verbe dans le cadre de la phrase alors que le constituant disloqué et coréférentiel (mon sac) se trouve dans une position topicalisée, soit à droite (3), soit à gauche (4). C'est la dislocation à gauche (4) qui nous intéresse dans ce travail. La dislocation à gauche ou à droite, existe également en norvégien. Tout comme en français le constituant disloqué du norvégien est annoncé (5) ou repris (6) par une forme pronominale dans le cadre de la phrase:

5) Jeg mistet den $i$ i dag morges, sekken min . $_{\text {. }}$ je perdis le ce jour matin, sac.DEF mon Je l'ai perdu ce matin, mon sac

6) Sekken min $_{i}$, den ${ }_{i}$ mistet jeg $i$ dag morges. sac.DEF mon, le perdis je ce jour matin Mon sac, je l'ai perdu ce matin.

Cependant, les propriétés des proformes dans les deux langues sont très différentes. Dans la classification translinguistique établie par Cardinaletti \& Starke (1999), on peut faire une tripartition des pronoms en formes fortes, faibles (légèrement déficientes) ou clitiques (fortement déficientes). Cette répartition suit une gradation hiérarchique des formes forte $>$ faible $>$ clitique suivant laquelle les formes faibles partagent certaines propriétés à la fois avec les formes clitiques et les formes fortes. Par exemple, une forme forte permet typiquement la coordination contrairement aux formes clitiques, tandis qu'une forme faible accepte parfois la coordination et parfois pas. La forme forte n'apparaît que dans des contextes spécifiques, par exemple comme complément d'une préposition ou dans des propositions elliptiques. Dans le système de la dislocation, la forme forte (moi) peut apparaître en position topicalisée avant d'être reprise par un pronom clitique attaché au verbe. La forme clitique peut coexister avec plusieurs autres formes clitiques (ne l') dans le nœud de la phrase: 
7) $M o i_{i}, j e_{i}$ ne l'ai pas perdu.

Pour la forme clitique, le français distingue trois cas (nominatif, accusatif et datif) à la troisième personne (du singulier et du pluriel), deux cas (nominatif et oblique) à la première et à la deuxième personne du singulier et une seule forme, applicable aux trois cas, à la première et à la deuxième personne du pluriel.

D’un point de vue morphologique, le système norvégien est moins complexe que son équivalent français, du moins pour la langue écrite. Notons que le norvégien, contrairement à la plupart des langues, y compris le français, possède deux variantes officielles à l'écrit: le bokmål (littéralement la «langue du livre») et le nynorsk (littéralement le «néo-norvégien»). Le nynorsk est une langue construite sur la base de dialectes du pays, conçue comme dénominateur commun de tout ce qui est typiquement norvégien dans la langue et qui s'oppose au bokmål, variété qui représente l'évolution de la langue en usage dans le pays sous l'influence danoise'. Qu'il s'agisse du bokmål ou du norvégien, à l'écrit, on ne fait aucune distinction entre formes forte, faible ou clitique:

8) $\mathrm{Jeg}_{i}$, jeg $g_{i}$ har ikke mistet den. (Bokmål)

je, je ai pas perdu le

Moi, je ne l'ai pas perdu.

9) $E g_{i}, e g_{i}$ har ikkje mista han. (Nynorsk)

je, je ai pas perdu le

Moi, je ne l'ai pas perdu.

À l'exception des variantes formelles entre les pronoms personnels de la première personne, la négation (ikke - ikkje) et la distinction à la troisième personne qui se fait uniquement en bokmål entre den (masculin, singulier, $3^{\mathrm{e}}$ personne, non humain) et han (masculin, singulier, $3^{\mathrm{e}}$ personne, humain), les pronoms norvégiens de l'écrit sont uniquement marqués pour le cas, nominatif ou oblique (saufle masculin à la $3^{\mathrm{e}}$ personne du singulier de nynorsk). Le pays possède cependant un grand nombre de dialectes qui jouissent d'un statut relativement privilégié et dont les traits morphosyntaxiques se distinguent des

1 La Norvège a été sous la domination danoise pendant plus de 350 ans, entre 1450 et 1814. Le bokmål est de loin la variante linguistique la plus importante, utilisée par presque $90 \%$ des écoliers dans le pays contrairement au nynorsk qui est utilisé dans une petite partie de l'ouest du pays. 
variantes standard de l'écrit. Dans certains dialectes, on trouve en effet les trois types de pronoms de la classification de Cardinaletti \& Starke (1999): distinction à la troisième personne du singulier, féminin, entre henner (forme forte), $h u$ (forme faible, nominative), henne (forme faible, oblique) et $a$ (forme oblique clitique). Et pour la troisième personne du singulier, celle du masculin, entre han (forme forte et faible) et $n$ (forme oblique clitique):

10) $\mathrm{Jeg}_{i}$, jeg $g_{i}$ ha'kke mista’n. (Norvégien parlé)

Je, je ai pas perdu le

Moi, je ne l'ai pas perdu.

Alors que les proformes du français (exemples 3, 4 et 7) sont toutes clitiques, leurs équivalents norvégiens ne peuvent jamais être à la forme clitique. On en trouve une illustration en (11) (tiré de Eide, 2011: 189) qui exige la forme faible $(h u)$ comme élément résomptif excluant la forme clitique $(a)$ :

11) Mamma hu/*'a var reingjøringskone. (Norvégien parlé) maman elle elle.CL était femme de ménage Maman elle était femme de ménage.

En ce qui concerne les formes clitiques sujet du français contemporain, leur statut est incertain. Tout comme en norvégien, l'élément topicalisé du français standard (12) est généralement traité comme un sujet disloqué, avec reprise pronominale clitique du sujet au sein de la phrase (13). Cependant, pour le français informel, dit avancé, on parle souvent d'un processus de grammaticalisation suivant lequel les proformes sont incorporées comme des marqueurs d'accord au verbe en laissant libre la place du sujet. Le sujet lexical topicalisé pourra ainsi être réanalysé comme le vrai sujet de la phrase (14):

12) $\mathrm{Lui}_{i} i_{i}$ dort

13) TTOPP $_{\text {Lui] }}\left[_{T P}[i l]\left[_{T 0}\right.\right.$ dort $\left.\left.]\right]\right]$.

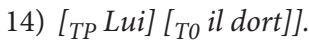

Pour argumenter en faveur de l'analyse affixale de (14) (ZribiHertz, 1994; Girard Lomheim, 2018), on note qu'il n'y a généralement pas de pause intonative entre le pronom tonique (lui) en position sujet et l'affixe ( $i l)$ attaché au verbe. Il y a également une tendance en français informel à omettre d'autres éléments clitiques, tels le ne clitique ou l'objet clitique, de la position préverbale. Pour un exemple comme (15) ci-dessous, il n'est pas possible de déterminer, hors contexte, si la 
forme clitique (ils ou $i$ ) est un marqueur d'accord dans une structure à redoublement du sujet (à comparer avec 14) ou une forme clitique résomptive dans une structure de dislocation à gauche:

15) Les enfants ils / i dorment.

Cependant, ce qui est clair, c'est que les structures en (13) et (14) sont toutes deux accessibles pour le locuteur francophone. Un sujet quantifié, on le sait (Girard Lomheim, 2018), ne peut être topicalisé:

16) ? Tout le monde, il est beau.

Avant de poursuivre nos remarques sur ces questions dans la section 3 ci-dessous, il nous importe d'introduire les données sur lesquelles nous baserons notre comparaison entre la topicalisation en français et en norvégien.

\section{La topicalisation en français et en norvégien}

Malgré les différences structurales entre les deux langues, le procédé de topicalisation existe aussi bien en français qu'en norvégien. Nous présenterons d'abord des cas avec la topicalisation du sujet avant d'aborder celle de l'objet, qu'il soit direct ou indirect, puis celle des adverbiaux.

\subsection{La topicalisation du sujet}

À première vue, la topicalisation du sujet semble obéir aux mêmes principes dans les deux langues en question. Qu'il s'agisse du français ou du norvégien, un groupe nominal disloqué à gauche peut être repris par une proforme résomptive reléguant le verbe à la troisième position de la phrase (V3):

17) Yoan $_{i} i_{i}$ aime le chocolat.

18) Johan ${ }_{i}$ han $_{i}$ liker sjokolade. Johan il aime chocolat Johan il aime le chocolat

Dans les deux cas, l'ordre inverse entre l'élément topicalisé et la forme résomptive est impossible: ${ }^{\star} I l$, Yoan.../ ${ }^{*}$ Han, Johan...

Les deux langues acceptent également la reprise d'un pronom topicalisé. En français, c'est la forme forte, non clitique, qui est excentrée avant d'être reprise par la forme clitique ou affixée au sein de 
la phrase, précédée ou non d'une pause intonative (Delais-Roussarie et al., 2004; De Cat, 2007a\&b). En norvégien, par contre, les deux formes pronominales sont fortes ou faibles, jamais clitiques, avec une pause séparant nécessairement les deux pronoms (Faarlund, 1992; Eide, 2011):

19) $M o i_{i}, j_{i}$ 'aime le chocolat.

20) $\mathrm{Jeg}_{i}$ jeg $\mathrm{g}_{i}$ liker sjokolade.

Moi j'aime chocolat

Moi j'aime le chocolat.

Malgré de telles différences sur le plan prosodique, on trouvera, pour la topicalisation du sujet, des structures V3 aussi bien en français qu'en norvégien. Rien de tel pour la topicalisation des objets.

\subsection{La topicalisation de l'objet direct}

Si l'objet direct est topicalisé, la proforme résomptive du français est attachée à gauche du verbe tout comme les autres formes clitiques:

21) Sandra ${ }_{i}$, je ne liai pas vue hier.

En (21), le pronom clitique objet direct à la forme accusative l' est précédé d'autres formes clitiques (en l'occurrence, le clitique sujet je et le ne clitique). Toutes les formes clitiques se succèdent, tandis que dans la structure norvégienne, le groupe nominal lexical (Sandra) doit précéder immédiatement le pronom résomptif:

22) Sandra ${ }_{i}$ henne $_{i} /$ hu $_{i}$ møtte jeg ikke i går.

Sandra elle elle recontrer.PRET je pas.NEG hier

Sandra je ne l'ai pas rencontrée hier.

Pour des raisons évidentes - le pronom clitique du français doit être attaché à la gauche du verbe - l'ordre entre le groupe nominal topicalisé et la proforme résomptive de (21) ne peut être changé: ${ }^{\star} L a$, je n'ai pas vu Sandra hier. Même avec une forme forte en position topicalisée, une telle permutation semble difficilement recevable: ${ }^{*}$ Elle, je n'ai pas vu Sandra hier. En principe, il en va de même pour le changement de l'ordre des éléments dans l'équivalent norvégien de (22): ${ }^{\star} H e n n e$, Sandra møtte jeg i går. Mais, dans ce cas, on pourrait imaginer une restructuration pragmatique du type: 
23) Henne , Sandra $_{i}$ altså, møtte jeg ikke i går.

elle, Sandra donc, recontrai.PRET je pas.NEG hier

Elle, c'est-à-dire Sandra, je ne l'ai pas rencontrée hier.

L'acceptabilité, quoique marginale de (23), indique que la permutation de l'ordre entre groupe nominal topicalisé et proforme résomptive est inacceptable pour des raisons pragmatiques plutôt que purement syntaxiques. Nous y reviendrons dans la section 3.

\subsection{La topicalisation de l'objet indirect ou datif}

Dans les deux langues, la topicalisation d'un objet indirect ou datif est possible, mais cette fois-ci des facteurs de complexité supplémentaires sont à considérer. Le français possède un datif (25) qui correspond au groupe prépositionnel de (24). La forme ne peut être utilisée après la préposition $\grave{a}$, sauf dans des contextes de contraste:

24) Sandra a donné un cadeau à son frère.

25) Sandra lui a donné un cadeau.

26) Sandra a donné un cadeau à lui (et non pas à elle).

Les mêmes possibilités existent en norvégien, mais les structures avec un groupe prépositionnel et un datif sont alors interchangeables:

27) Sandra ga en gave til broren sin.

Sandra donner.PRET un cadeau à frère.DEF son

Sandra a donné un cadeau à son frère.

28) Sandra ga han en gave.

Sandra donner.PRET lui un cadeau

Sandra lui a donné un cadeau.

29) Sandra ga en gave til han.

Sandra donner.PRET un cadeau à lui

Sandra lui a donné un cadeau.

On pourrait ainsi s'attendre à ce que la topicalisation de l'objet indirect datif de (27) implique la dislocation d'un groupe prépositionnel en til - «à»-, suivie d'une phrase qui contient le pronom datif. Ceci nous donnerait cependant des cas clairement agrammaticaux pour le norvégien comme en (30). La seule possibilité d'une reprise par un pronom est d'extraire le groupe nominal du groupe prépositionnel et de laisser la préposition in situ comme en (31). Par contre, si l'on ajoute la particule résomptive så - «alors» - après l'élément topicalisé, la 
phrase devient acceptable avec un groupe prépositionnel topicalisé (32) (Salvesen, 2020), mais demeure exclue avec la préposition orpheline (33). On sait également que le norvégien accepte assez librement la thématisation d'un groupe prépositionnel sans reprise résomptive (34). Notons que dans tous ces cas, le verbe se trouve dans la deuxième (V2) ou dans la troisième position (V3) de la phrase:

30) ${ }^{*}$ Til broren $\sin _{i}$, han $i$ ga Sandra en gave. à frère.DEF son, lui donner.PRET Sandra un cadeau Son frère, Sandra lui a donné un cadeau.

31) Broren $\sin _{i}$, han ${ }_{i}$ ga Sandra en gave til $-i$. frère.DEF son lui donner.PRET Sandra un cadeau à Son frère, Sandra lui a donné un cadeau.

32) Til broren sin, så ga Sandra en gave. à frère.DEF son, alors donna.PRET Sandra un cadeau À son frère, Sandra lui a donné un cadeau.

33) *Broren sin så ga Sandra en gave til_. frère.DEF son så Sandra donner.PRET un cadeau à À son frère, Sandra lui a donné un cadeau.

34) Til broren sin ga Sandra en gave. à frère.DEF son donna.PRET Sandra un cadeau À son frère Sandra a donné un cadeau.

La généralisation d'un $S \AA$ résomptif s'étend à d'autres types de complémentations indirectes, en l'occurrence au locatif:

35) Til Bergen så dro han alene. à Bergen alors aller.PRET il seul A Bergen il y est allé tout seul.

En français, par contre, le cas non marqué de la dislocation d'un datif ou d'un objet indirect est un groupe nominal en position topicalisée avec reprise pronominale du datif (36). Cependant, (36) ne représente pas la seule possibilité. Si le groupe prépositionnel entier est disloqué, Blasco-Dulbecco (1999) invoque la stratégie du double marquage (Delais-Roussarie et al., 2004; Berrendonner, 2015), d'où l'alternance entre (36) et (37):

36) Son frère, Sandra lui $i_{i}$ a donné un cadeau.

37) À son frère $e_{i}$ Sandra lui $i$ a donné un cadeau

Malgré le caractère marqué de (37) par rapport à (36) - il s'agit de $11,36 \%$ des cas chez Blasco-Dulbecco (1999: 115) - le choix d'avoir un 
groupe prépositionnel en position topicalisée existe pour le locuteur natif, que ce soit pour des datifs (37) ou des objets indirects:

38) De ce voyage, il s'en ${ }_{i}$ est longtemps souvenu.

C'est le cas aussi pour des objets indirects locatifs:

39) À Paris, il $y_{i}$ est allé hier.

40) Paris ${ }_{i}$, il $y_{i}$ est allé hier.

Le français a également une structure semblable à celle de son équivalent norvégien de (34) qu'on appelle "mouvement du focus» dans la tradition cartographique de Rizzi (1997). Cette fois-ci, le constituant topicalisé n'est pas repris par un clitique, emploi qu'on trouve essentiellement dans des contextes contrastifs, avec changement de thème:

41) À Simone j'ai donné un bouquet de fleurs. A Sandra, j’ai donné un livre...

Résumons-nous: en comparant les systèmes français et norvégien, nous avons constaté que les deux langues acceptent la topicalisation du sujet et de l'objet direct ou indirect. Quant aux objets indirects, datifs ou locatifs, le français a une tendance à topicaliser le groupe nominal sans préposition, alors que les conditions d'emploi en norvégien sont très différentes: si le groupe prépositionnel entier est topicalisé, il peut être repris par la particule $S \AA ̊$. Il est également possible de topicaliser le complément de la préposition en laissant la préposition in situ. C'est uniquement dans ce dernier cas que la reprise pronominale est possible, et ce dans une position suivant directement l'élément topicalisé. Le verbe quant à lui reste dans la deuxième ou la troisième position de la phrase. Dans la section suivante, nous essaierons d'expliquer en termes plus théoriques les différences observées entre les deux systèmes.

\section{Propositions théoriques}

Le contraste entre (38) et (39) ci-dessus indique très clairement que la périphérie gauche $\mathrm{du}$ français contient plusieurs positions thématiques. Avec ce point de départ, nous aborderons les différences structurales entre le français et le norvégien dans une perspective plus théorique. 


\subsection{Cadre théorique}

A ses débuts, la grammaire générative percevait la périphérie gauche de la phrase comme une projection du complémenteur COMP, qui allait former plus tard un groupe complémenteur CP (voir par exemple Lasnik \& Lohndal, 2013). Les analyses plus fines de Pollock $(1989 ; 1997)$ pour l'architecture du domaine verbal de la phrase (IP ou TP) et celles de Rizzi (1997) pour la périphérie gauche de la phrase (CP) ont permis aux analyses cartographiques de voir le jour. Selon les propositions originales de Rizzi (1997), l'éclatement du CP nous donnait des positions fixes pour des projections fonctionnelles étagées à la périphérie gauche:

$$
\text { Force ... Top } \text { T. .. Foc ... Top }^{*} \ldots \text { Fin ... }
$$

Le modèle de Rizzi permettait des positions topiques récurrentes, symbolisées par Top ${ }^{*}$, soit précédant, soit suivant la projection de Focus. Cette analyse fut remplacée dans Benincà \& Poletto (2004) par un schéma plus rigide selon lequel les topiques étaient divisés en un champ FRAME et un champ THEME qui, tous deux, précédaient le FOCUS :

FRAME ... THEME ... FOCUS

Ce qui nous intéresse particulièrement ici, c'est que le cadre (ou le FRAME) contient deux positions: des Hanging Topics (HT), ce que Berrendonner (2015) appelle des «nominatifs pendants» et des adverbes scéniques, alors que la position thématique (THEME) se divise en topiques disloqués à gauche (Left Dislocation - LD) et en topiques interprétés comme des listes (List Interpretation Topic LIT). Benincà \& Poletto (2004) ne parlent pas explicitement de la position de Force, mais elles semblent supposer que Force sépare les deux champs des topiques qui tous deux précèdent les positions de Focus et de FinP:

[FRAME Hanging Topic ... Scene Setting] ... Force ...

[THEME Left Dislocation Topic ... List Interpretation Topic] ... Focus ... FinP ...

Notons que le modèle de Benincà \& Poletto (2004: 64-65) se base sur des données de l'italien. Il ne s'applique donc pas per se au français et au norvégien. Par exemple, l'italien étant une langue à pro-drop, le sujet résomptif peut être implicite, ce qui n'est pas le cas pour le français où le clitique sujet (ou le sujet affixé) doit être exprimé. Contrairement au français et à l'italien, le norvégien a uniquement des proformes 
résomptives faibles et non-clitiques. Malgré de telles différences, nous retiendrons une observation importante de l'approche de Benincà \& Poletto (voir également Delais-Roussarie et al., 2004): il y a au minimum deux positions pour les topiques à la périphérie gauche de la phrase, l'une pour le «nominatif pendant» (HT) et l'autre pour le topique disloqué à gauche (LD). Et si la périphérie gauche contient deux éléments topicalisés, le nominatif pendant précède le topique disloqué à gauche.

\subsection{Propriétés des topiques du français}

En se basant sur le système de Cinque (1983), Delais-Roussarie et al. (2004: 504-505) soutiennent qu'il y a lieu de maintenir la division binaire des LD et des HT pour le français. Leur argument principal est que les expressions topicalisées dans (44) et (45) n'obéissent pas aux mêmes restrictions d'îlot (islands constraints):

42) ${ }^{\star}$ A Marie $_{i}$, je connais le flic qui lui $i_{i}$ a retiré son permis

43) Marie ${ }_{i}$, je connais le flic qui lui $i_{i}$ retiré son permis.

Suivant cette analyse, le groupe nominal topicalisé Marie de (43) est nécessairement un HT ayant la même référence que le pronom datif lui. Ceci s'explique par le fait qu'un HT est par définition une expression nominale alors que le LD garde le même marquage de Cas que l'élément résomptif. Si bien que le groupe prépositionnel de (42) doit correspondre à une dislocation à gauche (LD). L'agrammaticalité de cette structure s'expliquerait par le fait que la subordonnée relative du français constitue une configuration d'îlot d'où le pronom datif ne saurait s'échapper. La dislocation à gauche serait ainsi dérivée par mouvement de l'élément disloqué vers la gauche, ce qui est impossible en (42). Selon Delais-Roussarie et al., il s'agit alors en (43) d'un HT généré directement dans sa position disloquée de base. Le HT a la même référence que le pronom datif de la relative sans risque de violer la contrainte d'îlot: ainsi, l'élément résomptif peut être enchâssé sans être dérivé par mouvement. Une telle argumentation est cependant contredite par le fait que le français accepte plusieurs éléments disloqués à gauche (Delais-Roussaire et al., 2004: 505; Lambrecht, 2001). Il s'agit dans (44) de deux HT (Marie, ce crime), alors que (45) 
illustre la présence de deux LD (À Marie, de ce crime). Rappelons que les groupes prépositionnels de (45) constituent par définition des LD:

44) Marie, ce crime, je crois que je ne lui en parlerai pas.

45) À Marie, de ce crime, je crois que je ne lui en parlerai pas.

Il est également possible, selon les mêmes auteurs, de combiner des HT avec des LD à la périphérie gauche pourvu que le HT précède le LD:

46) Marie, de ce crime, je crois que je ne lui en parlerai jamais.

47) ?À Marie, ce crime, je crois que je ne lui en parlerai jamais.

48) Ce crime, à Marie, je crois que je ne lui en parlerai jamais.

49) ?De ce crime, Marie, je crois que je ne lui en parlerai jamais.

La grammaticalité de (46) et de (48) s'explique par le fait que les HT (Marie, ce crime) précèdent les LD (de ce crime, à Marie). Dès qu'on inverse l'ordre des deux types de topiques, le résultat est moins acceptable (cf. (47) et (49)). Sur la base de tels exemples, on pourra donc inférer que le français a deux types de topiques, d'une part des $\mathrm{HT}$, d'autre part des LD, et que s'il y a à la fois un HT et un LD dans la phrase, le HT précède le LD. Mais s'il y a plusieurs éléments topicalisés, on voit difficilement comment ces éléments topicalisés seraient dérivés par mouvement.

En effet, De Cat (2007a\&b) conteste les analyses par mouvement indépendamment de la distinction entre le HT et le LD. Les informateurs du français oral de De Cat (2007b: 499-500) acceptent largement des exemples comme (50), (51) et (52), qui contiennent tous ce qu'on définit généralement comme des "îlots» (la subordonnée interrogative de (50), la subordonnée adverbiale de (51) et le groupe prépositionnel de (52):

50) Aux petits ${ }_{i}$, je sais pas [ce [qu'elle leur ${ }_{i}$ lit]].

51) Ta mère $e_{i}$ je ferai tout pour être parti [quand elle ${ }_{i}$ viendra].

52) Aux autres; ${ }_{i}$ on va attendre [avant de leur ${ }_{i}$ parler].

Il s'agit, dans le système de Roussarie-Delais et al., d'un LD dans (50) et (52) (aux petits; aux autres) et, sans doute, d'un HT (ta mère) dans (51). De Cat, quant à elle, refuse les analyses par mouvement pour tous ces exemples. Quoi qu'il en soit, il nous manque des critères sûrs pour décider si l'on est en présence de HT ou de LD sans ancrage pragmatique ou prosodique distinct entre les deux types de 
dislocations. Comment peut-on savoir s'il s'agit dans (53) d'un HT ou d'un LD (exemple type de Roussarie-Delais et al., 2004: 501)?:

53) Cet homme $e_{i}$ je ne le $e_{i}$ connais pas.

S'il n'y a aucune différence fondamentale entre le HT et le LD, comme le veut De Cat, il faudrait également savoir pourquoi le constituant disloqué le plus à droite dans les exemples (46) et (48) ci-dessus, à savoir les LD tels que les définissent Roussarie-Delais et al. et Lambrecht, est plus étroitement lié à son élément résomptif dans la phrase (doté du même marquage de cas) que le constituant disloqué le plus à gauche, dit HT, où il n'y a aucune ressemblance de cas entre l'élément topicalisé et la proforme résomptive.

Il semble donc qu'il y ait des arguments solides pour le français contre les analyses par mouvement des constituants disloqués à gauche. Ceci est particulièrement le cas pour le HT et cela pourrait être le cas également pour le LD (De Cat, 2007a\&b). Considérons maintenant les différences entre les structures du français et du norvégien.

\subsection{Propriétés des topiques du norvégien}

Le norvégien accepte assez facilement, on l'a vu, des LD du type (54) On y trouve également des structures qui ressemblent fortement à des HT (55):

54) Den filmen, den ${ }_{i}$ husker jeg ikke. ce film.DEF, ce souviens je pas Ce film, je ne m'en souviens pas.

55) Foreldra mine $i$, jeg var sikker på at du kjente dem (Faarlund 1992: 120) parents.DEF mes, je étais sûr à que tu connaissais les.ACC Mes parents, j'étais sûr que tu les connaissais.

En (54), l'objet direct disloqué est repris par le pronom personnel inanimé den qui suit directement l'élément topicalisé sans qu'il y ait nécessairement de pause entre les deux éléments. En (55), par contre, il y a nécessairement une pause importante après le constituant disloqué. On pourrait même imaginer, à l'écrit, la marque d'une césure pourvue d'une force illocutionnaire après le constituant topicalisé: point d'interrogation (56), particule ja «oui» (57). Et contrairement à 
la dislocation à gauche, l'élément résomptif ne suit pas nécessairement le constituant topicalisé:

56) Foreldra mine $e^{\text {? Jeg var sikker på at du kjente dem }} i$ (Faarlund, 1992: 120)

parents.DEF mes je étais sûr à que tu connaissais les.ACC Mes parents, jeétais sûr que tu les connaissais.

57) Foreldra mine $i$, ja, jeg var sikker på at du kjente dem $i$ parents.DEF mes oui je étais sûr à que tu connaissais les.ACC Mes parents, jeétais sûr que tu les connaissais.

Ainsi parvient-on à distinguer le HT de (56) et (57) et le LD de (58) où l'élément résomptif suit directement le LD:

58) Foreldra mine , dem $_{i}$ var jeg sikker på at du kjente. parents.DEF mes les.ACC étais je sûr à que tu connaissais Mes parents, jeétais sûr que tu les connaissais.

En adoptant les principes d'analyse de Faarlund (1992) et d'Eide (2011), on avancera que la pause intonative nous permet d'identifier des HT et des LD également pour le norvégien. Ceci dit, une pause peut aussi être envisagée pour le LD de (54). Notons à cet égard que les formes clitiques nominales du norvégien parlé - $n$ ' pour le masculin et $a^{\prime}$ pour le féminin - demeurent exclues après le constituant topicalisé:

59) Den filmen ${ }_{i}{ }^{*} n /$ den $_{i}$ husker jeg ikke.

ce film ce.CL ce souviens je pas

Ce film, je ne m'en souviens pas.

De tels faits semblent indiquer la présence d'une pause intonative aussi bien pour le LD que pour le HT, mais montrent aussi que la force illocutionnaire d'une telle pause ne saurait être valide que pour le HT comme l'indiquent (56) et (57). De plus, pour le LD, l'élément résomptif suit obligatoirement l'élément topicalisé, ce qui n'est pas nécessairement le cas pour le HT. Il y a également des restrictions très fortes sur la co-occurrence de plusieurs topiques à la périphérie gauche en norvégien. On n’y trouve, de préférence, qu'un seul élément disloqué.

Sur la base de telles données, on argumente souvent en faveur d'une analyse par mouvement du constituant disloqué en norvégien, comme pour les langues germaniques en général (Grohmann, 2000; Frascarelli \& Hinterhölzl, 2007). Dans de tels modèles d'analyse, les restrictions sur les possibilités de dislocations multiples dans les langues germaniques 
sont également liées aux propriétés des langues V2. Donc, on ne pourrait pas avoir plusieurs constituants qui passent par une seule position topicalisée. On ajoutera que le norvégien accepte des cas où le déterminant possessif apparaît dans la position topicalisée:

60) Bilen $\sin _{i}$, den tar Per $i$ godt vare $p a ̊ a$. voiture.DEF POSS. REFL celle prend Per bien soin de Sa voiture, il s'en occupe bien.

Le déterminant possessif $\sin$ (masculin, singulier) doit être lié, dans une relation de c-commande, par le sujet nominal de la phrase Per, ce qui n'est pas possible dans sa position (topicalisée) de surface. Autrement dit, si l'on exige que le constituant topicalisé soit lié par le sujet, il peut l'être comme complément de la préposition $p a ̊$ «à » dans sa position de base. Il s'ensuit que le LD de (60) a été dérivé par mouvement.

Ce qui est encore plus intéressant, c'est que le groupe nominal topicalisé contenant un possessif peut également précéder la particule ja «oui» dont la présence est considérée par Eide (2011), rappelons-le, comme un critère du HT:

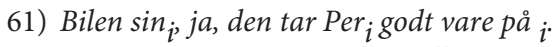
voiture.DEF POSS.REFL oui elle prend Per bien soin de Sa voiture, il s'en occupe bien.

Il semblerait donc que, pour le norvégien, il faudrait accepter une analyse par mouvement même pour le HT, ce qui a pour implication que le HT du norvégien peut avoir un marquage de Cas. En effet, l'idée que le HT ait été déplacé concorde avec l'opinion de Boeckx \& Grohmann (2004) qui aboutissent à la même conclusion pour l'allemand.

\section{Conclusion}

Dans cet article, nous avons vu que le français comme le norvégien acceptent la topicalisation de sujets, d'objets directs et d'objets indirects, locatifs ou datifs. En ce qui concerne la dislocation du sujet, l'élément résomptif suit le constituant topicalisé dans les deux langues, mais le français possède deux structures formellement identiques: sujet disloqué à gauche et redoublement du sujet. Pour la dislocation des objets (directs ou indirects), des différences formelles séparent les deux langues: l'élément résomptif du norvégien suit en principe toujours le 
constituant topicalisé contrairement à son équivalent clitique français qui est attaché, comme toute forme clitique, à gauche du verbe. Avec les objets indirects, il est également possible en norvégien de reprendre le constituant topicalisé par la particule så laissant le verbe dans la troisième position. Il est intéressant de noter que les deux langues acceptent des groupes nominaux pleins comme objets indirects topicalisés. En français, cette structure est de loin privilégiée même si on trouve également des groupes prépositionnels topicalisés, repris par des clitiques. Le norvégien peut recourir à des prépositions orphelines qui nous permettent de topicaliser le complément de la préposition.

Nous avons vu qu'il est légitime, pour les deux langues, de conserver la division "classique» entre deux types de topiques: le nominatif pendant $(\mathrm{HT})$ et la dislocation à gauche (LD). Cette division s'établit formellement en français dans la mesure où le HT d'un objet indirect a la forme d'un groupe nominal plein, alors que le LD garde le marquage prépositionnel. Il est également possible, dans certaines occurrences, d'exclure la dérivation par mouvement quand des structures d'enchâssement viennent bloquer les opérations d'extraction. Pour le norvégien, nous avons à la fois des critères syntaxiques et prosodiques pour distinguer les deux types de topicalisations. Le HT est marqué par une pause, et il peut également avoir une force illocutionnaire indépendante. De cette façon, nous pouvons nous baser sur le schéma cartographique de Benincà \& Poletto (2004) pour situer les HT et les LD dans la structure syntaxique. Le HT est par conséquent associé à une autre position que le $\mathrm{LD}$, position précédant celle de FORCE. Celui-ci est plus étroitement lié au contenu de la phrase, ce qui explique le comportement distinct des HT et des LD aussi bien en français qu'en norvégien:

[FRAME Hanging Topic ... Scene Setting] ... Force ... [THEME Left Dislocation Topic ... List Interpretation Topic] ... Focus ... FinP ...

Nous avons établi que les LD du norvégien peuvent être dérivés par mouvement, associant une telle opération syntaxique au statut du norvégien comme langue V2. La dérivation par mouvement pourrait même s'appliquer pour le HT. En français, par contre, les dérivations s'opèrent sans mouvement. Le français est une langue non-V2 et la possibilité de multiplier des constituants disloqués à la périphérie gauche existe aussi bien pour les HT que pour les LD (Lambrecht, 
2001; Delais-Roussarie et al., 2004; De Cat, 2007a\&b). Autrement dit, contrairement au norvégien, les constituants topicalisés du français sont générés directement dans leur position de base, à la périphérie gauche de la phrase.

\section{Bibliographie}

BENINCÀ P. \& POLETTO C. (2004), Topic, focus, and V2, in Rizzi L. (ed.), The Structure of CP and IP: The Cartography of Syntactic Structures, Oxford, Oxford University Press, 52-75.

BERRENDONNER A. (2015), Constructions disloquées, in Encyclopédie grammaticale du français, en ligne: encyclogram.fr

BLASCO-DULBECCO M. (1999), Les dislocations en français contemporain: Étude syntaxique, Paris, Honoré Champion.

BOECKX C. \& GROHMANN K. (2004), Left dislocation in Germanic, in Abraham W. (ed.), Focus on Germanic Typology, Berlin, Akademie Verlag, 131-44.

CARDINALETTI A. \& STARKE M. (1999), The typology of structural deficiency: A case study of the three classes of pronouns, in van Riemsdijk H. (ed.), Clitics in the Languages of Europe, Berlin, Mouton de Gruyter, 145-234.

CINQUE G. (1983), Topic constructions in some European languages and connectedness, in Ehlich K. \& van Riemsdijk H. (eds.), Connectedness in Sentence, Discourse and Text, Tilburg, Hogeschool Tilburg, 7-41.

DE CAT C. (2007a), French dislocation, interpretation, syntax, acquisition, Oxford, Oxford University Press.

DE CAT C. (2007b), French dislocation without movement, Natural Language and Linguistic Theory 25, 485-534.

DELAIS-ROUSSARIE E., DOETJES J. \& SLEEMAN P. (2004), Dislocation, in de Swart H. \& Corblin F. (éds), Handbook of French Semantics, Stanford, CSLI Publications, 501-529.

EIDE K.M. (2011), Norwegian (non-V2) declaratives, resumptive elements, and the Wackernagel position, Nordic Journal of Linguistics 34, 179-213.

FAARLUND J.T. (1992), Norsk syntaks i funksjonelt perspektiv, Oslo, Universitetsforlaget.

FRASCARELLI M. \& HINTERHÔLZL R. (2007), Types of topics in German and Italian, in Schwabe K. \& Winkler S. (eds.), On 
Information Structure, Meaning and Form, Amsterdam, John Benjamins, 87-116.

GIRARD LOMHEIM F. (2018), Le pronom dans le français de Louisiane, Paris, L'Harmattan.

GROHMANN K. (2000), Copy left dislocation, in Billerey R. \& Lillehaugen B. D. (eds.), WCCFL 19 Proceedings, Somerville, MA, Cascadilla Press, 139-152.

HOLMBERG A. (2015), Verb Second, in Kiss T. \& Alexiadou A. (eds.), Syntax - Theory and Analysis, Berlin, Mouton de Gruyter, 34283.

LAMBRECHT K. (2001), Dislocation, in Haspelmath M. (éd.), Language typology and language universals: an international handbook, Berlin, De Gruyter, 1050-1078.

LASNIK H. \& LOHNDAL T. (2013), Brief Overview of the History of Generative Grammar, in den Dikken M. (ed.), The Cambridge Handbook of Generative Syntax, Cambridge, Cambridge University Press, 26-60.

POLLOCK J. (1989), Verb Movement, Universal Grammar, and the Structure of IP, Linguistic Inquiry 20, 365-424.

POLLOCK J. (1997), Langage et cognition, Paris, Presses Universitaires de France.

RIZZI L. (1997), The fine structure of the left periphery, in Haegeman L. (ed.), Elements of Grammar. A handbook of generative syntax, Dordrecht, Kluwer, 281-337.

ROWLETT P. (2007), The Syntax of French. Cambridge, Cambridge University Press.

SALVESEN C.M. (2020), Adverbial resumptive particles and Verb Second, in Woods R. \& Wolfe S. (eds.), Rethinking Verb Second, Oxford, Oxford University Press.

ZRIBI-HERT, A (1994), La syntaxe des clitiques nominatifs en français standard et avancé, Travaux de linguistique et de philologie 32, 131-147. 